\title{
OPTIMAL EXPERIMENTAL DESIGN FOR MODELING BATTERY DEGRADATION
}

\author{
Joel C. Forman \\ Mechanical Engineering \\ University of Michigan \\ Ann Arbor, Michigan 48109 \\ jcforman@umich.edu \\ Jeffrey L. Stein \\ Mechanical Engineering \\ University of Michigan \\ Ann Arbor, Michigan 48109 \\ stein@umich.edu
}

\author{
Scott J. Moura \\ Mechanical and Aerospace Engineering \\ University of California, San Diego \\ San Diego, California 92093 \\ smoura@ucsd.edu \\ Hosam K. Fathy* \\ Mechanical and Nuclear Engineering \\ Pennsylvania State University \\ University Park, Pennsylvania 16802 \\ hkf2@psu.edu
}

\section{ABSTRACT}

Accurate battery health modeling allows one to make better design decisions, enables health conscious control, and allows for feed-forward State of Health estimation. However, experiments are necessary in order to obtain and validate these models. Unfortunately, battery health experiments are costly in terms of time, person-hours, and equipment. This makes it extremely important to minimize the number of experimental iterations.

This paper aims to minimize time and expense of experiments while maximizing information gathered by bridging an important gap between the Optimal Experimental Design (OED) and the battery health experimental/modeling literature. We demonstrate how to apply static OED methods to a battery aging experiment. This allows us to select a set of Constant Current Constant Voltage (CCCV) cycles that maximizes the amount of information gathered - in turn allowing us to better identify the health model parameters. The CCCV cycling is carried out in a laboratory using $14 \mathrm{LiFePO}_{4}$ cells (10 for fitting and 4 for validation). Each of these cells undergoes 429 days of battery health cycling. Results from these experiments include: a model of battery capacity fade based on voltage and current, battery health dependence on voltage, and a lack of power fade under the cycling conditions. The use of OED to coordinate our model form and experiment helped to ensure a fruitful model resulted when processing the collected data. Based on this success we suggest a

\footnotetext{
*Address all correspondence to this author.
}

generalized framework for Optimal Battery Health Model Experiments (OBHME), which allows one to apply OED to a variety of related problems.

\section{NOMENCLATURE}

$F \quad$ Fisher Information Matrix [Various]

$H$ Battery Capacity [Various]

I Battery Current [Amp]

$I_{+} \quad$ CCCV Charge Current Limit [Amp]

I_ CCCV Discharge Current Limit [Amp]

$I_{\text {trickle }}$ CCCV Trickle Current Limit [Amp]

$P \quad$ Battery Power [Watt]

$U$ Trial Matrix [Various]

$V \quad$ Battery Terminal Voltage [Volt]

$V_{\text {min }}$ CCCV Minimum Voltage [Volt]

$V_{\max } \mathrm{CCCV}$ Maximum Voltage [Volt]

$f(\cdot)$ Experiment Regressor Function [Various]

$i$ Model Parameter Index [Index]

$j \quad$ Experiment Index [Index]

$k$ Trial Index [Index]

$m$ Number of Parameters [Unitless]

$n$ Number of Trials [Unitless]

$p \quad$ Number of Possible Trials [Unitless]

$q$ Number of Trials in Experiment [Unitless]

$t_{f}$ Duration of Repeated Cycle [Sec]

$t_{\text {hold }}$ CCCV Float Hold Time [Sec] 
$u$ Experiment Regressors [Various]

$\Xi \quad$ All Possible Experiments [Set]

$\alpha$ Optimized Trial Index [Index]

$\beta$ Model Parameters [Various]

$\lambda \quad$ Trial Time Fraction [Unitless]

$\sigma$ Standard Deviation of Measurement Noise [Various]

\section{INTRODUCTION}

In recent years there has been increasing interest in vehicle electrification. Electrification has the potential to both decrease green house gas production and improve energy independence. However, to realize these benefits, Plug-in Hybrid Electric Vehicle (PHEVs) must have battery packs that do not rapidly degrade. Accurate modeling of battery health can help both manage and mitigate battery degradation in a variety of ways. Battery health modeling allows for more intelligent PHEV design decisions regarding battery size and drive train topology, helping to minimize cost and improve reliability. Battery health modeling also enables health conscious control, which extends battery life through judicious real-time management of battery and engine utilization [1]. In addition, battery health modeling enables feed-forward State of Health ( $\mathrm{SoH}$ ) estimation, improving online estimates of available battery power and capacity. Clearly, accurate battery health modeling is a critical tool for designing and controlling PHEV battery packs. However, battery health modeling for PHEVs is a challenge due to the aggressive nature of battery pack use.

This challenge emphasizes the importance of experiments for both obtaining and validating battery health models. However, conducting battery health experiments can be extremely costly in terms of time, person-hours, and equipment; efficient use of experimental resources is critical. This paper aims to maximize information gained subject to constraints on experiment time and expense by bridging an important gap between the Optimal Experimental Design (OED) and battery experimental $/$ modeling literatures.

To this end, we investigate a specific case of designing and conducting a battery health modeling experiment using 14 $\mathrm{LiFePO}_{4}$ cells. This experiment is optimally designed to identify model parameters under given experimental resource constraints. Results include: a model of battery capacity fade based solely on voltage and current data, demonstration of battery health dependence on voltage, and a lack of power fade (at $100 \%$ State of Charge, SoC) under the cycling conditions. The success of this case suggests a generalization: the Optimal Battery Health Model Experiment (OBHME) framework. This framework allows one to apply static OED methods to a large class of battery health modeling experiments. OBHME uses Fisher information and the Cramér-Rao bound to optimally guide the selection of experimental trials. We believe this case and associated framework show the broad applicability and utility of OED when considering battery health modeling.
The remainder of this paper is organized as follows. A literature review follows the introduction, giving a focused review of both $\mathrm{LiFePO}_{4}$ battery health experiments and a general overview of OED. This is followed by a section which applies OED to health modeling for PHEV type batteries. This case involves both a theoretical OED part regarding applying static OED to dynamic health models and an experimental part in which the batteries undergo laboratory cycling. Experimental results follow, beginning with model fitting and continuing to general observations regarding the degradation of these battery cells. The OBHME framework follows this, generalizing the procedure and suggesting alternative design choices. A discussion section follows, focusing on specific experimental details we would modify in future experiments based on experience gained. The final section presents several conclusions drawn from this work.

\section{LITERATURE REVIEW \\ $\mathrm{LiFePO}_{4}$ Battery Health Experiments}

While many groups have conducted battery health experiments, we focus on two that use the same $\mathrm{LiFePO}_{4}$ battery type as in this paper's case study [2,3]. In [2] the batteries undergo $\mathrm{CCCV}$ cycling with varying current rates, depths of discharge, and temperatures. They fit a health model to the experimental data, dependent on charge processed, temperature, and maximum C-rate. This model provides useful insights (especially regarding temperature effects), but is not control oriented due to the inclusion of maximum $\mathrm{C}$-rate as a parameter. A second group cycles these batteries under conditions related to PHEV drive cycles [3]. Five different cycles with drive and Vehicle-toGrid (V2G) portions are taken as a set of representative behavior. Their analysis of the experiments results in a control oriented model that is a function of energy processed and scalar that depends on if the cell is undergoing driving or V2G. Our paper improves on the existing experimental methods by demonstrating the applicability of OED to battery health experiments.

\section{Overview of Optimal Experimental Design}

The OED literature is broadly divided into experiment design for static and dynamic models. This paper focuses on the static case and here we introduce the reader to relevant literature. A classic OED text is that of Fedorov [4]. An excellent first introduction to OED is presented by Atkinson, Donev, and Tobias which covers nearly all that the practitioner would need to design an experiment [5]. For a more in depth study of Fisher information and the Cramér-Rao bound one can examine Cover and Thomas, which does an excellent job presenting these mathematical concepts [6]. A thorough discussion of optimization of the nonlinear case is presented by Walter and Pronzato [7]. OED provides a variety of mathematical tools for improving results and decreasing expense of model based experiments. 


\section{BATTERY HEALTH MODELING EXPERIMENT}

This section presents an optimal battery health modeling experiment for $\mathrm{LiFePO}_{4}$ cells. A later section discusses the OBHME framework - a generalization of this specific case. This case and the general framework share the same major steps: model selection, experiment design, and realization. In model selection one chooses a health metric (output), input(s), and form. This model guides the experiment design, which selects trials based on optimizing information gathered regarding the model parameters. Experiment design involves considering what trials are possible, generating and regressing their input trajectories, and using an optimization to select an experimental trial set. In realization, the laboratory experiment collects data which is then used to estimate the model parameters. The remainder of this section discusses the experiment and introduces a variety of concepts related to the OBHME framework.

\section{Model Selection}

Battery Health Metric For this experiment two different battery health metrics are considered: capacity fade and power fade. For PHEV applications capacity fade is related to how far the vehicle can drive without charging and power fade is related to maximum available electric power. In this paper's experimental section we discuss measuring these metrics in a laboratory. For the modeling work later in the paper we focus exclusively on the capacity fade health metric as conclusive power fade has not been observed in our experimental work.

Choice of Model Inputs Battery health can depend on a wide variety of factors. However, for this work our goal is to create a control oriented battery health model. Thus, we focus solely on two factors readily accessible to battery management systems: voltage and current. This choice of inputs ensures that the model will be applicable to many real-time control applications. Our interest in voltage is largely due to it being a proxy for battery SoC. We would have used SoC directly, however, it is difficult to accurately and robustly regulate battery $\mathrm{SoC}$ during long term health experiments. As an alternative we use voltage as an input and add longer time holds $(1800 \mathrm{sec})$ to the float charge/discharge segments of the CCCV cycles. These time holds allow the battery to better equilibrate towards the relaxed open circuit voltage, which is closely related to SoC. As will be shown later, the use of voltage and current as inputs allows one to make reasonable predictions about battery degradation.

Battery Health Model Form The inputs and output are connected by the model form. An effective model form considers a priori knowledge (if available) in addition to the model's intended application. In this work a black box model is used due to uncertainty in the underlying degradation process, leading to a model form based on regression. This regression form is based on our specific interest in the battery health dependence on voltage and current polarity. This potential voltage dependence is interesting because it affects optimal PHEV charge scheduling (scheduling charging at different times changes the PHEV battery rest voltage). The effect of current polarity is important because the battery degradation rate may change during charging and discharging.

Based on these interests and a desire to keep the number of model parameters small we arrived at the following model form, referred to as the "Asymmetric" model:

$$
\begin{aligned}
\dot{h}\left(I_{+}, I_{-}, V\right) & =\beta_{1}+\beta_{2} I_{+}+\beta_{3} I_{-}+\beta_{4} V+\beta_{5} I_{+}^{2}+\beta_{6} I_{-}^{2} \\
& +\beta_{7} V^{2}+\beta_{8} I_{+} V+\beta_{9} I_{-} V+\beta_{10} V^{3} .
\end{aligned}
$$

Where $I_{+}$is the current charging the cell, $I_{-}$is the current discharging the cell, $V$ is the cell terminal voltage, and $h$ is the battery health. This model has different behavior in charging versus discharging, a cubic dependence on voltage, and can age under zero current conditions.

To keep our exposition more abstract we focus on a generalized form of the model in Eq. 1:

$$
\dot{h}(\vec{x})=\sum_{i=1}^{m} \beta_{i} f_{i}(\vec{x}) .
$$

Where $f_{i}$ maps the model inputs $\vec{x}$ to real numbers. This model form is Linear in its Parameters (LP) and has no autoregressive component. An additional model of this form, referred to as the "Symmetric" model, is:

$$
\begin{aligned}
\dot{h}(I, V) & =\beta_{1}+\beta_{2}\|I\|+\beta_{3} V+\beta_{4}\|I\|^{2}+\beta_{5} V^{2} \\
& +\beta_{6}\|I\| V+\beta_{7} V^{3}
\end{aligned}
$$

Where, unlike Eq. 1, this model does not distinguish between current polarities. With the model in Eq. 1 selected, the experiment is now ready to be optimized.

\section{Experiment Design}

Possible Trial Set Trial selection is the key to optimizing experiments. This selection begins with the set of all possible trials, $\Xi$. For battery health experiments members of $\Xi$ are typically rules for cycling an individual battery for a period of time. We use CCCV cycling to generate a wide variety of robust inputs for long term cycling. These CCCV cycles are described by the archetype cycling in Alg. 1. Three tunable parameters are used to construct $\Xi: V_{\min }, V_{\max }$, and $I_{\max }$, with values:

$$
\begin{aligned}
V_{\min } & \in\{2.0 \mathrm{~V}, 2.1 \mathrm{~V}, \cdots, 3.5 \mathrm{~V}\} \\
V_{\max } & \in\{2.1 \mathrm{~V}, 2.2 \mathrm{~V}, \cdots, 3.6 \mathrm{~V}\} \\
I_{\max } & \in\{0.5 \mathrm{C}, 1.0 \mathrm{C}, \cdots, 2.5 \mathrm{C}\} .
\end{aligned}
$$




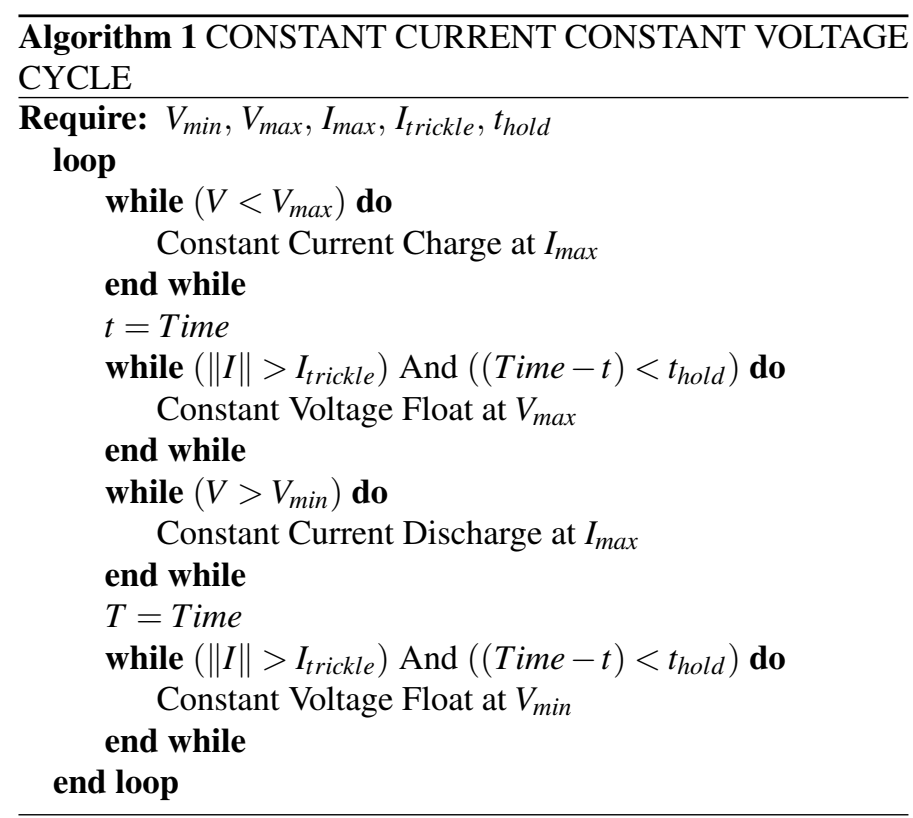

This results in a total of 680 unique cycles. The next step of experiment design uses these rules to generate (approximate) input trajectories.

Input Trajectory Generation Input trajectories are generated using electrochemical battery simulation. Specifically, we simulate voltage and current trajectories with the DoyleFuller-Newman (DFN) cell model [8-10]. The parameter values of this model are obtained from a previous study that fit the DFN model to a $\mathrm{LiFePO}_{4}$ battery [11]. To better approximate quasi steady-state conditions, the third cycle simulated is used. In spite of identical charge and discharge current limits, the battery exhibits asymmetric behavior in charging and discharging, due to its electrochemical nature. Next, we regress these input trajectories, encapsulating their data for the optimization algorithm.

Input Trajectory Regression Regressor vectors encapsulate how trial input trajectories affect the estimation information gathered. This encapsulation converts the dynamic form of the model into a static form appropriate for optimization. Since the health measurements happen intermittently we only collect discrete measurements of change in health over a time interval. Consider integrating the model over the time period between health tests:

$$
\begin{aligned}
\int_{0}^{t_{f}} \dot{h}(\vec{x}) d t & =\int_{0}^{t_{f}} \sum_{i=1}^{m} \beta_{i} f_{i}(\vec{x}) d t \\
\Delta h & =\sum_{i=1}^{m} \beta_{i} u_{i},
\end{aligned}
$$

where:

$$
\begin{aligned}
\Delta h & \equiv \int_{0}^{t_{f}} \dot{h}(\vec{x}) d t \\
u_{i} & \equiv \int_{0}^{t_{f}} f_{i}(\vec{x}) d t .
\end{aligned}
$$

Here $\Delta h$ is the change in battery health and each $u_{i}$ is a regressor associated with the function $f_{i}$. Unique trials can be indexed with $j$ and the model can be rewritten as follows:

$$
\Delta h_{j}=\sum_{i=1}^{m} \beta_{i} u_{i j}=\vec{\beta} \cdot \vec{u}_{j} .
$$

This specific form will be important for the optimization algorithm. For now it provides a formula to compute the $\vec{u}_{j}$ regressor vectors:

$$
\vec{u}_{j}=\left(\int_{0}^{t_{f}} f_{1}(\vec{x}(t)) d t, \cdots, \int_{0}^{t_{f}} f_{m}(\vec{x}(t)) d t\right)^{T} / t_{f}
$$

These regressor vectors will form the rows of the Fisher information matrix which will be optimized through the selection of experimental trials.

Experiment Optimization Let us consider a natural way to compile the experimental data. One can create a matrix equation by stacking each $\Delta h_{j}$ into a vector of health measurements and stacking each $\vec{u}_{j}^{T}$ into a corresponding matrix row:

$$
\left(\begin{array}{c}
\Delta h_{1} \\
\Delta h_{2} \\
\vdots \\
\Delta h_{n}
\end{array}\right)=\left[\begin{array}{cccc}
u_{11} & u_{12} & \cdots & u_{1 m} \\
u_{21} & u_{22} & \ddots & \vdots \\
\vdots & \ddots & \ddots & \vdots \\
u_{n 1} & \cdots & \cdots & u_{n m}
\end{array}\right] \vec{\beta} .
$$

This can be rewritten as:

$$
H=U \vec{\beta}
$$

where $H$ and $U$ are the appropriate matrices.

The Fisher information matrix $F$ is then defined as:

$$
F=\frac{U^{T} U}{\sigma^{2}},
$$

where the measurement error of experiment is assumed be a normal distribution with zero mean and variance $\sigma^{2}$. The inverse of $F$ is the best possible covariance one can achieve when estimating $\vec{\beta}$ due to the Cramér-Rao bound [6]. Furthermore by using 
least squares, an unbiased estimator, this bound is achieved. Thus the covariance in estimating $\vec{\beta}$ is:

$$
\operatorname{covar}(\vec{\beta})=\sigma^{2}\left(U^{T} U\right)^{-1}
$$

The importance of Eq. 12 is twofold. Firstly it shows that the response of the trials does not affect the covariance for estimating $\vec{\beta}$ so it can be ignored for the experimental design (this occurs because of the LP assumption). Second one can influence the covariance directly through the selection of experimental trials. For our work we assume that $\sigma^{2}$ is fixed, but in practice one can influence this value as well, by using better sensors or testing methods. We now describe the DETerminant MAXimizing algorithm (DETMAX), an optimization method for selecting experimental trials.

The objective of OED is to select trials that allow one to estimate $\vec{\beta}$ with minimum covariance (by a given metric). Doptimum is a very common metric in OED and is based on maximizing the determinant of the Fisher information matrix. This in turn, minimizes the determinant of its inverse, the covariance matrix. Minimizing the determinant of the covariance matrix minimizes the product of its eigenvalues. Geometrically if one investigates the confidence region of the $\vec{\beta}$ estimate this results in the smallest possible (by content) hyper ellipsoid [5].

DETMAX attempts to find a subset of fixed size $q$ contained in $\Xi$ that maximizes the value of $\operatorname{det}(F)$. It does this by selecting regressor vectors from $\Xi$ to create the experiment matrix $U$. Mathematically DETMAX attempts to:

$$
\begin{aligned}
& \max _{\vec{\alpha}} \operatorname{det} \frac{U^{T} U}{\sigma^{2}} \\
& U=\left[\begin{array}{llll}
\vec{u}_{\alpha_{1}} & \vec{u}_{\alpha_{2}} & \cdots & \vec{u}_{\alpha_{q}}
\end{array}\right]^{T},
\end{aligned}
$$

subject to:

$$
\begin{aligned}
& \alpha_{i} \in\{1, \cdots, p\} \\
& i \neq j \Rightarrow \alpha_{i} \neq \alpha_{j} \\
& \vec{u}_{i} \in \Xi
\end{aligned}
$$

DETMAX attempts to select locally D-optimum trial subsets contained in $\Xi$ from the set of all possible experiments. Here local optimality is in the sense of swapping - one cannot swap any of the experiments in the set with any other possible experiment and increase $\operatorname{det}(F)$. In practice we initialize this algorithm with many random sets to help ensure that the resulting set is closer to the global optimal. DETMAX has a few variations, the version we use is as follows. First DETMAX computes $\operatorname{det}(F)$ for the current set. Then it iterates over all possible swaps of trials between this set and the set of all possible experiments. For each swap a new $\operatorname{det}(F)$ is computed and stored. The swap
Table 1. EXPERIMENTAL TRIALS AS DETERMINED BY DETMAX

\begin{tabular}{clll}
\hline Batch & $V_{\min }$ & $V_{\max }$ & $I$ \\
\hline 1 & $3.0 \mathrm{~V}$ & $3.3 \mathrm{~V}$ & $2.5 \mathrm{C}$ \\
1 & $2.6 \mathrm{~V}$ & $3.6 \mathrm{~V}$ & $1.5 \mathrm{C}$ \\
1 & $2.0 \mathrm{~V}$ & $3.5 \mathrm{~V}$ & $0.5 \mathrm{C}$ \\
1 & $3.0 \mathrm{~V}$ & $3.1 \mathrm{~V}$ & $2.5 \mathrm{C}$ \\
1 & $3.2 \mathrm{~V}$ & $3.5 \mathrm{~V}$ & $2.5 \mathrm{C}$ \\
1 & $2.0 \mathrm{~V}$ & $2.1 \mathrm{~V}$ & $0.5 \mathrm{C}$ \\
1 & $2.4 \mathrm{~V}$ & $2.5 \mathrm{~V}$ & $0.5 \mathrm{C}$ \\
1 & $2.2 \mathrm{~V}$ & $3.5 \mathrm{~V}$ & $2.5 \mathrm{C}$ \\
1 & $2.0 \mathrm{~V}$ & $3.2 \mathrm{~V}$ & $2.0 \mathrm{C}$ \\
1 & $3.5 \mathrm{~V}$ & $3.6 \mathrm{~V}$ & $0.5 \mathrm{C}$ \\
\hline 2 & $3.4 \mathrm{~V}$ & $3.6 \mathrm{~V}$ & $2.5 \mathrm{C}$ \\
2 & $3.0 \mathrm{~V}$ & $3.4 \mathrm{~V}$ & $2.5 \mathrm{C}$ \\
2 & $2.0 \mathrm{~V}$ & $3.1 \mathrm{~V}$ & $2.5 \mathrm{C}$ \\
2 & $2.0 \mathrm{~V}$ & $3.6 \mathrm{~V}$ & $1.5 \mathrm{C}$ \\
\hline
\end{tabular}

which improves $\operatorname{det}(F)$ the most is then performed and the process repeats. DETMAX concludes when no swaps improve on $\operatorname{det}(F)[5,7]$.

DETMAX is ideal because we have a low number of battery cells and do not want to complicate the experiment by switching cycles after each health test. We decided to batch the experiment using 10 cells for the first batch and 4 cells for the second batch (DETMAX can be used to optimize batched experiments) $[5,7]$. The second batch is used solely for validation and is not included in the model fitting exercise. With the experimental trials selected our attention now turns to laboratory experiments.

\section{Realization}

Laboratory Experiment The batteries are repeatedly cycled based on the optimized set of trails (see Tab. 1). Thus far, the experiment has been carried out for 429 days with health data being collected approximately every two weeks. For health, we measure capacity fade as discharge capacity in Amp-Hours during $0.5 \mathrm{C} \mathrm{CCCV}$ cycling. These cycles repeat four times during each health test and the first value is removed to avoid memory (hysteresis) effects. Power fade is measured by a $2.5 \mathrm{C}$ constant current draw for 15 seconds when the batteries are fully charged. All of the tests are conducted at ambient room temperature. We set the cycle parameters $I_{\text {trickle }}$ to $50 \mathrm{~mA}$ and $t_{\text {hold }}$ to $1800 \mathrm{sec}$ onds. The cycling and health measurements are conducted using an Arbin BT-2000 battery cycler. 
This version of the cycler has 32 independent channels that can each source or sink up to 15A. Each of these channels can operate from $0 \mathrm{~V}$ to $10 \mathrm{~V}$ (but not negative). 14 of the 32 channels were dedicated to this experiment. All of the cycling was conducted at room temperature.

The cells cycled are manufactured by A123 Systems; the model number is ANR18650M1A. These cells are interesting because they are scaled down versions of those intended for use in PHEVs and are able to continuously discharge at 30C giving them excellent rate capability. They have a rated capacity of 1.1 Amp-Hours, a nominal voltage of $3.3 \mathrm{~V}$, and a voltage range from $2.0 \mathrm{~V}$ to $3.6 \mathrm{~V}$.

Equipment down time is addressed by back filling voltage data with constant values (current is taken as zero during these periods). This is an approximation, but since there was a limited amount of downtime and the batteries relax within a few hours this was considered appropriate.

As the battery experiment is conducted the model input trajectories (voltage and current) are recorded for each battery. This data is mapped to the regressor vectors associated with changes in battery health. These, along with the health data, are then used to calculate the parameters for the battery health model. In the next section we discuss the specifics of parameter estimation.

Parameter Estimation With all of the experimental data recorded estimating $\vec{\beta}$ is straightforward. One begins by compiling the recorded the health measurements and associated regressor vectors. These can then be arranged to form the matrix equation in Eq. 9. This, in turn, can be rewritten as Eq. 10, leading to the least squares solution of $\vec{\beta}$ as follows:

$$
\vec{\beta}=\left(U^{T} U\right)^{-1} U^{T} H
$$

The next section discusses the specific results of this model fitting work.

\section{EXPERIMENTAL RESULTS Model Fitting Results}

Our plan for this model fitting work was to use both power and capacity fade as health metrics. However, as is discussed in the next subsection, power fade was negligible during the experiment (at least for our method of measurement). Thus, we only consider the capacity health metric. As mentioned before this experiment divides the cells into two batches, the first for identification and the second for validation (the batches are conducted concurrently). Only data collected from the first batch is used in the estimation of $\vec{\beta}$.

Initially we attempted to fit the Asymmetric model, given in Eq. 1. We then considered the Symmetric model, given in Eq. 3 , which differs from the Asymmetric model only by not distinguishing between positive and negative current. It was found
Table 2. ESTIMATED PARAMETERS FOR THE SYMMETRIC MODEL

\begin{tabular}{|c|c|c|}
\hline Parameter & Value & Units \\
\hline$\beta_{1}$ & $1.2126 \times 10^{-7}$ & $A m p \times H o u r \times S e c^{-1}$ \\
\hline$\beta_{2}$ & $-3.1430 \times 10^{-8}$ & Hour $\times \mathrm{Sec}^{-1}$ \\
\hline$\beta_{3}$ & $-1.3861 \times 10^{-7}$ & Amp $\times$ Hour $\times$ Sec $^{-1} \times$ Volt $^{-1}$ \\
\hline$\beta_{4}$ & $2.5287 \times 10^{-10}$ & $A m p \times H o u r \times S e c^{-1} \times A m p^{-1}$ \\
\hline$\beta_{5}$ & $5.2377 \times 10^{-8}$ & Amp $\times$ Hour $\times$ Sec $^{-1} \times$ Volt $^{-2}$ \\
\hline$\beta_{6}$ & $8.2662 \times 10^{-9}$ & Hour $\times \operatorname{Sec}^{-1} \times$ Volt $^{-1}$ \\
\hline$\beta_{7}$ & $-6.5166 \times 10^{-9}$ & Amp $\times$ Hour $\times$ Sec $^{-1} \times$ Volt $^{-3}$ \\
\hline
\end{tabular}

that the Symmetric model predicts the validation slightly better than the Asymmetric model, which indicates that not all of the parameters in the Asymmetric model are needed for prediction. Figure 1 presents the Symmetric model prediction results for the validation cycles. These predictions are initialized with the first set of collected health data and then simulated open-loop. Figure 2 summarizes the prediction errors for the validation cycles with a histogram and percentile plot. Next we consider additional experimental observations that are not directly related to model fitting.
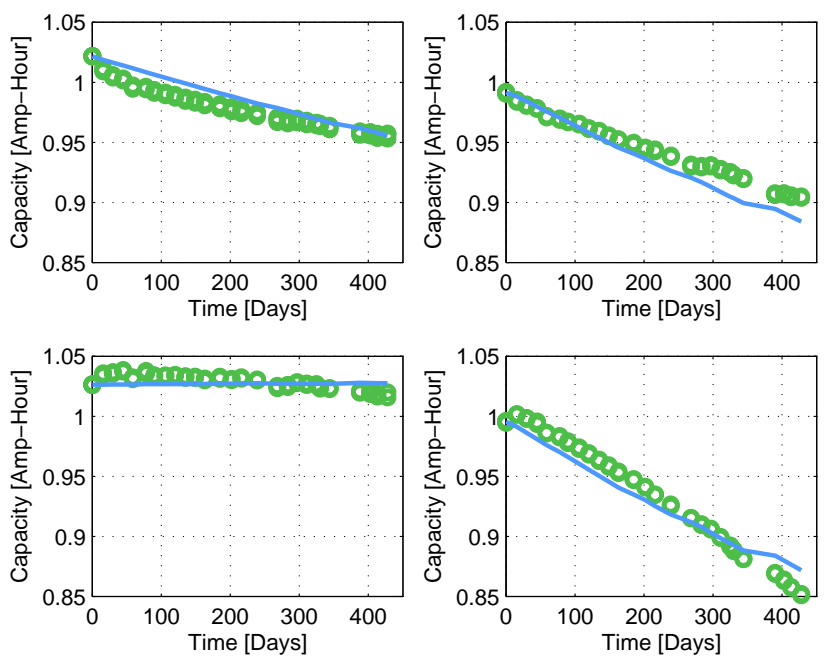

Figure 1. SYMMETRIC MODEL PREDICTIONS OF VALIDATION CYCLES. CURVE IS THE SYMMETRIC MODEL AND CIRCLES REPRESENT EXPERIMENTAL HEALTH MEASUREMENTS. 


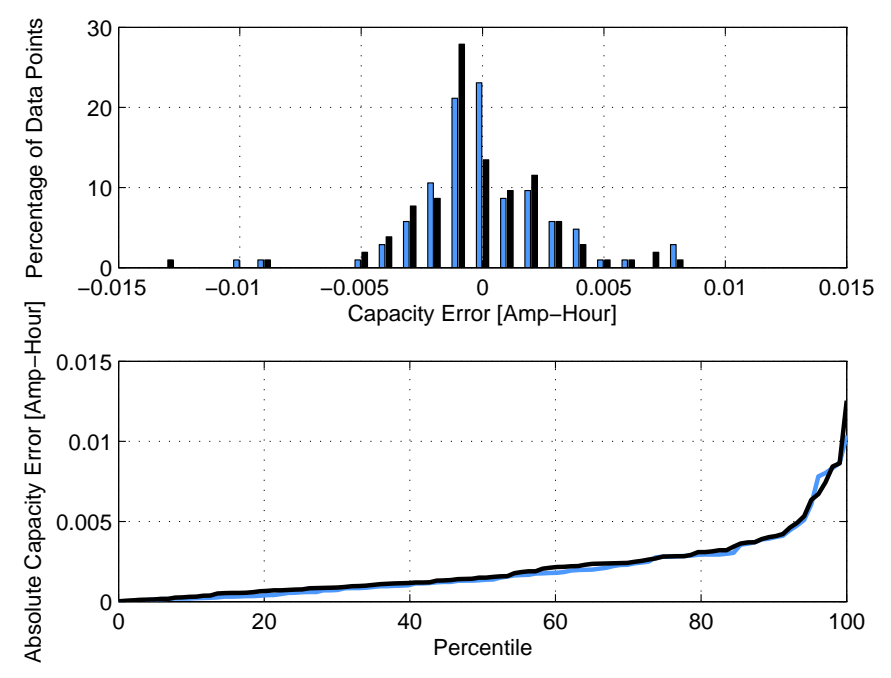

Figure 2. AGGREGATED ERRORS OF VALIDATION DATA WHEN USING THE SYMMETRIC MODEL FOR PREDICITON. ASYMMETRIC IN BLACK, SYMMETRIC IN BLUE.

\section{Results Independent of Model Fitting}

This section presents two experimental results that are independent of model fitting. The first is the dependency of battery aging on voltage, in contrast to [3], where battery degradation is found to be entirely a function of energy processed. Specifically batteries that undergo light duty cycling at voltages at or above $3.4 \mathrm{~V}$ age much more quickly than those cycled with voltages at $3.1 \mathrm{~V}$ or below. For the group of batteries that underwent more moderate cycling there is an approximately linear fit between battery health and energy processed - in agreement with [3]. Capacity results for these three groups are in presented Fig. 3.
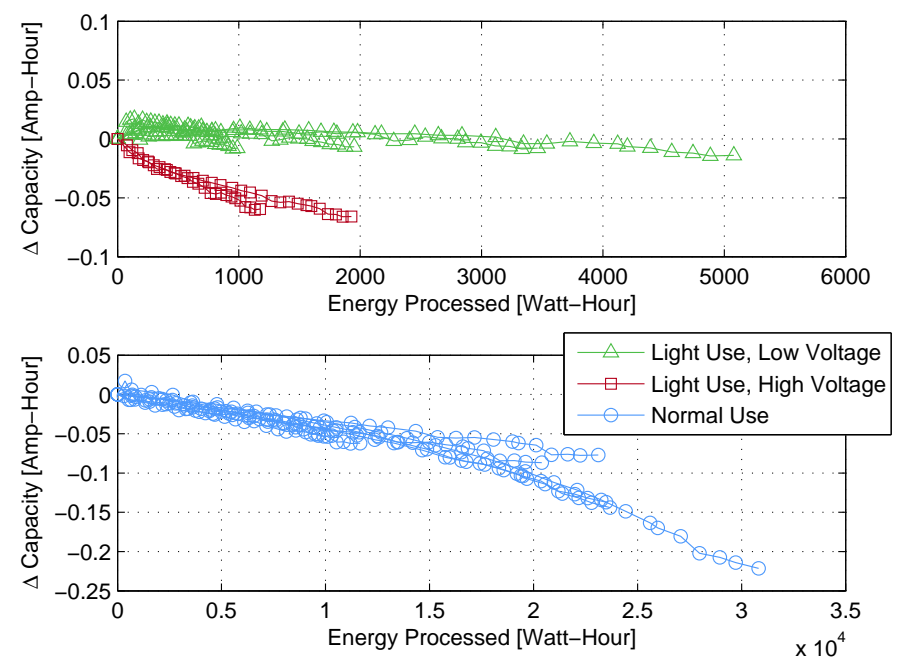

Figure 3. $\triangle$ CAPACITY AS A FUNCTION OF CHARGE PROCESSED
The second major result is that power fade is negligible in all of the batteries, see Fig. 4. This could perhaps be an artifact of either our measurement method or simply not driving the batteries aggressively enough. The batteries are rated for $30 \mathrm{C}$ discharge whereas they were tested at $2.5 \mathrm{C}$. The power test was a 15 second constant current draw, starting with the battery at $100 \%$ $\mathrm{SoC}$ (based on float charging at 3.6V). The recorded voltages and currents during this period were used to compute average power. Experimental design methods likely facilitated the appearance of the voltage dependence related to battery health. In the next section we discuss a generalization of this experimental work: OBHME.

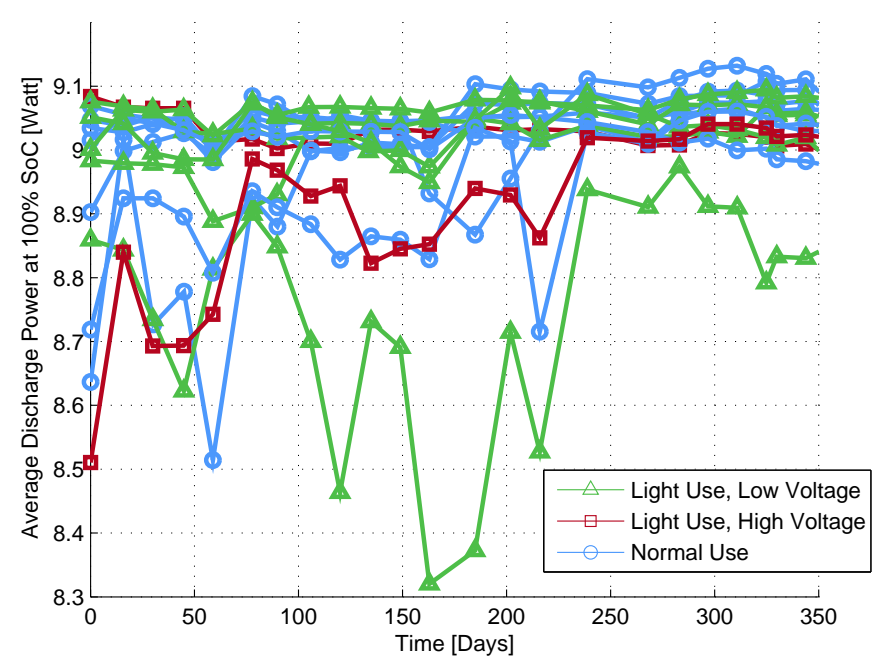

Figure 4. AVERAGE DISCHARGE POWER AS A FUNCTION OF EXPERIMENTAL TIME

\section{THE OBHME FRAMEWORK}

The work in this paper suggests a generalized framework for conducting optimal battery health modeling experiments. The OBHME framework has three main steps: model selection, experiment design, and realization; see Fig. 5. The first step, model selection, chooses health metrics, model inputs, and an appropriate model form. The second step, experiment design, creates the possible trial set, generates and regresses the associated trials, and optimizes the trial set to maximize Fisher information. The final step, realization, conducts the battery experiment and then estimates the model parameters. The remainder of this section highlights how OBHME provides a method appropriate for optimizing a wide variety of battery health experiments. 


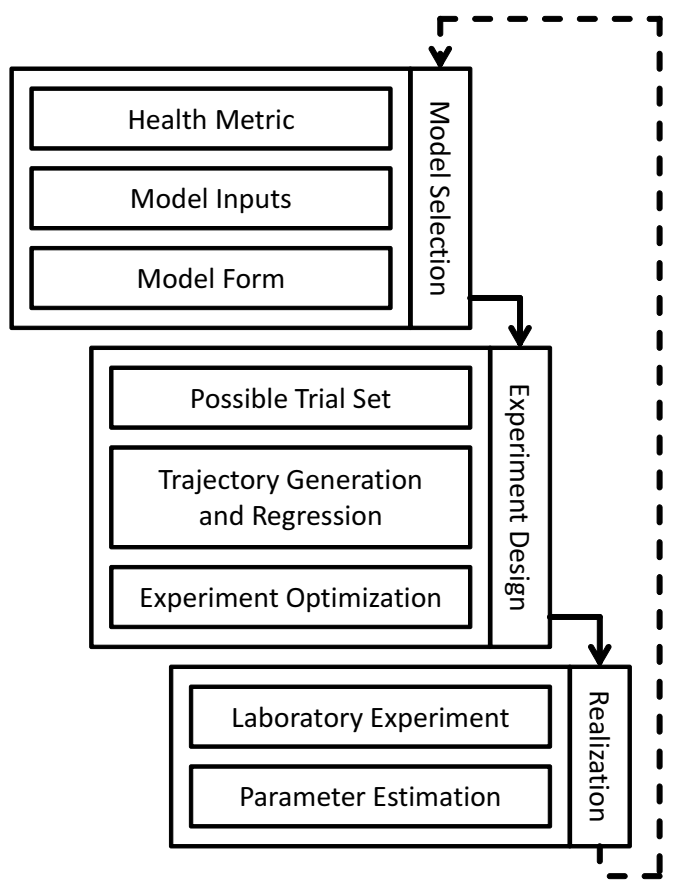

Figure 5. THE OBHME FRAMEWORK

\section{Model Selection}

The goal of OBHME is to efficiently produce an accurate model of observed physical phenomena. Typically, this model is intended for design, control, and/or optimization applications. When selecting a model one must consider the physical phenomena being investigated and the intended use of said model. The model selection step is critical because the optimal experiment depends directly on the selected model. The remainder of this section focuses on the individual parts of the battery health model: health metric (output), input(s), and model form.

Health Metric The battery health metric's importance is two-fold; it both measures battery health and quantifies the health model's output. Capacity and power fade are typical choices for health metrics, although a variety of additional metrics exist. For example, in destructive testing, one may consider looking at specific internal features of the battery such as the solid electrolyte interface layer's thickness. One may also use Electric Impedance Spectroscopy (EIS) to investigate the frequency response characteristics related to battery aging. While many features of battery health are of interest, a good metric considers the following factors: intended model application, physical phenomena, and measurement cost. Health metrics play key roles in both measuring aging phenomena and interpreting the battery health model's output.

Model Inputs Similar to model outputs, choice of model inputs is tremendously important for both observing phenomena and ensuring the model's later applicability. One may choose from a variety of inputs, including: voltage, current, $\mathrm{SoC}$, and temperature. Use of dynamic modeling and/or in situ measurements allows one to include various internal battery states as inputs. For example, local inputs such as current density, over potential and SoC can be used as inputs by using the DFN model to simulate them. Internal temperature can be included through the use of in situ temperature measurement [12]. The input and output of the battery health model are connected by its mathematical form.

Model Form The model form is selected based on a priori information if available, or an appropriate regression form otherwise. Models that can be cast in the form of Eq. 2 can be used without further mathematical complications. However, model's with autoregressive components or that are nonlinear in their parameters can still be accommodated in principle, but the mathematical complication will increase [7]. In the next step OBHME optimizes the experiment's trial set to gather the maximum parameter estimation information.

\section{Experiment Design}

The key to OBHME is deciding on a model before conducting the experiment and optimizing the selected experimental trials. The experiment design step breaks down into four steps. First one must decide on what set of experimental trials they are willing to consider - the possible trial set. Second one most generate their associated trajectories - the ones that will be input into the battery health model. These trajectories are then regressed into a form that makes them appropriate for parameter estimation. Finally an optimization method is used on these regressed forms to either select a subset or duty cycle fraction. The remainder of this section discusses alternative choices one can make when design these experiments.

Possible Trial Set The possible trial set $\Xi$ is the set of all trials one wishes to consider and can actually conduct for an experiment. Typically this is limited by available equipment and conditions desired for the battery modeling. Earlier we focused entirely on a set of CCCV cycles described by an archetype cycle. Here we present an alternative archetype cycle which would be based on PHEV drive cycles, the Drive Charge Cycle (DCC). The $\Xi$ s generated by DCC is interesting because they mimic what PHEV batteries would experience in the field. For driving the battery undergoes a current trajectory (that may involve charging and discharging) mimicking PHEV battery pack behavior with appropriate scaling. One can imagine a probabilistic type cycle parameterized by driver aggressiveness and trip length. Additionally the vehicle battery may be parameterized by size and weight when converting the drive cycles into current trajectories for the battery. After each drive (or several drives) the battery will need to be charged. This can be done using the charge portion of a CCCV cycle or one could use a Constant Power Constant Voltage (CPCV) cycle in order to better emulate battery 
pack charging. Additionally one can choose to emulate charging the vehicle with the various classes of PHEV home charging units. Algorithm 2 outlines how one would implement the DCC type cycles in a laboratory environment.

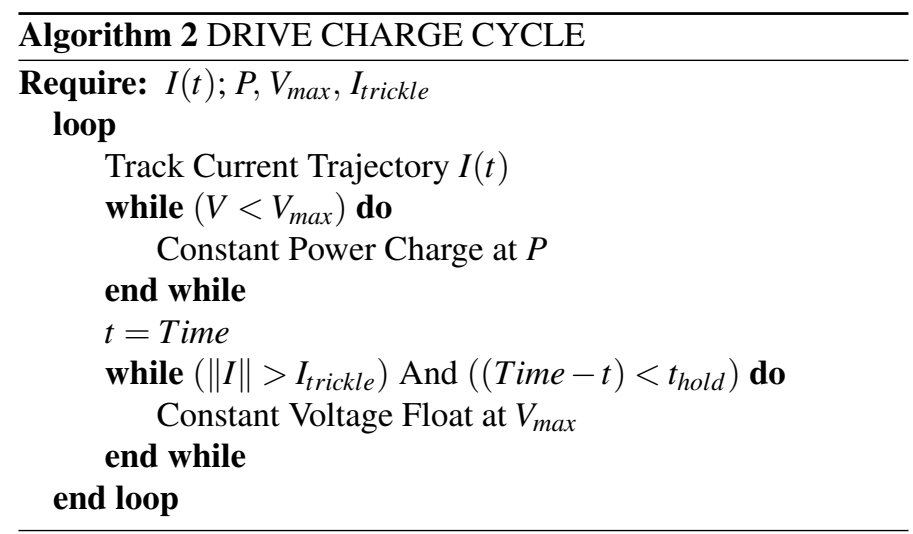

Of course, DCC is just one of the many possibilities. The possible trial set can be customized for cycles based on cell phones, laptops, and satellites. In the next section we consider how to convert these cycle rule sets into these input trajectories for the battery health model.

Trajectory Generation and Regression With a set of possible trials decided upon the trials must now be converted from rule sets to input trajectories for the health model. To achieve this one can generate quasi steady-state cycling trajectories through a variety of methods (these are cycles that repeat almost identically). The assumption here is that the input trajectories are not going to change substantially throughout the experiment, so all of the cycling behavior can be approximated in this behavior (alternative methods are needed for models with autoregressive components).

These quasi steady-state input trajectories are obtained in two main ways, both with tradeoffs. The first method is to use simulation. This requires that one has models that generate accurate trajectories of the variables to be used as inputs. An alternative method is to use laboratory equipment and directly measure the quasi steady-state cycles. One advantage of this method is it may result in highly accurate cycles as it eliminates model error. The results of both methods are the same, namely they both produce input trajectories from steady-state cycles based on trial rule sets. The choice between these methods is largely dependent on the size of $\Xi$ and available resources.

The input trajectories for each trial are then mapped to a trial regressor vector, to create a encapsulated version of the input trajectory's effects on the battery health model. The regression vectors are then collected into a set that describes the information available from various combinations of each of the trials. The procedure is the same as demonstrated earlier, see Eq. 8. Now we have a form amenable to optimizing the choice of experimental trials.

Experiment Optimization OBHME allows for the direct use of either the DETMAX algorithm or a Linear Matrix Inequality Interior Point algorithm (LMIIP) both of which provide optimal experimental designs given slightly differing experimental structures $[7,13]$. DETMAX was discussed in detail earlier so we will focus on LMIIP here.

Instead of selecting a small subset of trials for the experiment, as DETMAX does, LMIIP allows one to incorporate all the possible trials. Specifically LMIIP finds the global optimum of the following problem:

$$
\begin{aligned}
& \max _{\vec{\lambda}} \operatorname{det} \frac{U^{T} U}{\sigma^{2}} \\
& U^{T} U=\sum_{k=1}^{p}\left(\lambda_{k} \vec{u}_{k} \vec{u}_{k}^{T}\right),
\end{aligned}
$$

subject to:

$$
\begin{aligned}
& \sum_{k=1}^{p} \lambda_{k}=1 \\
& \lambda_{k} \geq 0, i=1, \cdots, p .
\end{aligned}
$$

Where here there are two interpretations. The first is that there are a large number of cycles conducted and the $\lambda_{i}$ s determine the portion of time that each cycle is being conducted. Alternatively, one can treat this as a probability mass function and use it to generate randomized subset of cycles [13]. This is especially nice for stochastic type cycles (such as the aforementioned DCC) because it allows the incorporation of stochasticity into the experiment. With the trials/time fractions selected we continue onwards to the laboratory experiment.

\section{Realization}

The battery experiment is carried out by repeatedly running the cycles associated with the optimized set of trials. This cycling is interrupted at various times to conduct battery health measurements as related to the previously selected metric. For experiments generated with LMIIP there must be a mechanism to change the cycles after every health measurement. The regressors associated with these health measurements are computed by mapping the recorded input trajectories in accordance with the model form (see Eq. 8). This data can then be directly compiled into a form amenable for the linear least squares estimation of $\vec{\beta}$ (see Eq. 15). At the conclusion of the method one may wish to design a new experiment based on the results, perhaps to fine tune the modeling efforts further. 


\section{DISCUSSION}

Based on experience gained from this experiment, we suggest several opportunities for improving future iterations. First, duplicating trials would help to better elucidate underlying statistical properties of the batteries. Second, for reasons mentioned earlier, future modeling efforts may be further improved through nonidentical current limits, increasing the charge and discharge asymmetry. Additionally, there are several areas where increasing experimental scope is also desirable. For example, temperature can become an additional input by using several thermal chambers to regulate multiple ambient temperatures. Also, more aggressive cycling is possible by increasing the current limits, leading to a health model with broader applicability (although internal temperature may need to be considered). Further, replacing $\mathrm{SoC}$ as an input for voltage could provide additional insights into battery degradation, at the expense of requiring a robust implementation strategy. Finally, power testing could be augmented by conducting it at different SoCs, higher rates, and/or incorporating EIS. Clearly, the experience gained from this experiment suggests several advantageous improvements and extensions.

\section{CONCLUSIONS}

This paper demonstrates how to apply OED to a specific battery case and then introduces a general framework for handling a wide variety of battery health experiments. This specific case designs and conducts a battery health modeling experiment for $\mathrm{LiFePO}_{4}$ battery cells. The experiment results in a control oriented model useful for design, control, and optimization of PHEVs. This initial work suggests the OBHME framework, which bridges an important gap between the OED literature and the battery health experimental/modeling literature. It is hoped that the OBHME framework case study in this paper provides a guide for making battery health experiments less costly in terms of time, effort, and equipment and more profitable in terms of information and model accuracy.

\section{ACKNOWLEDGMENT}

The authors would like to acknowledge the NSF-EFRI Grant \#0835995 for financial support and the DOE-ARRA for providing financial support for laboratory equipment.

\section{REFERENCES}

[1] Moura, S. J., Forman, J. C., Bashash, S., Stein, J. L., and Fathy, H. K., 2011. "Optimal Control of Film Growth in Lithium-Ion Battery Packs via Relay Switches”. Industrial Electronics, IEEE Transactions on, 58(8), aug., pp. 3555 $-3566$.

[2] Wang, J., Liu, P., Hicks-Garner, J., Sherman, E., Soukiazian, S., Verbrugge, M., Tataria, H., Musser, J., and Finamore, P., 2011. "Cycle-Life Model for GraphiteLiFePO4 Cells”. J. Power Sources, 196, pp. A260-A271.
[3] Peterson, S. B., Apt, J., and Whitacre, J., 2010. "Lithiumion Battery Cell Degradation Resulting from Realistic Vehicle and Vehicle-To-Grid Utilization”. J. Power Sources, 195, pp. 2385-2392.

[4] Fedorov, V., 1972. Theory of Optimal Experiments. Probability and mathematical statistics. Academic Press.

[5] Atkinson, A., Donev, A., and Tobias, R. Optimum Experimental Designs, with SAS. Oxford statistical science series.

[6] Cover, T., and Thomas, J. Elements of Information Theory. Wiley series in telecommunications.

[7] Walter, É., and Pronzato, L., 1997. Identification of Parametric Models from Experimental Data. Communications and control engineering. Springer.

[8] Doyle, M., Fuller, T., and Newman, J., 1993. "Modeling of Galvanostaic Charge and Discharge of the Lithium/Polymer/Insertion Cell”. J. Electrochemical Society, 140, June, pp. 1526-1533.

[9] Fuller, T., Doyle, M., and Newman, J., 1994. "Simulation and Optimization of the Dual Lithium Ion Insertion Cell". J. Electrochemical Society, 141, January, pp. 1-10.

[10] Forman, J., Bashash, S., Stein, J. L., and Fathy, H. K., 2010. "Reduction of an Electrochemistry-Based Li-Ion Battery Model via Quasi-Linearization and Pade Approximation". J. Electrochemical Society, 158(2), December, pp. A93A101.

[11] Forman, J., Moura, S., Stein, J. L., and Fathy, H. K., 2011. "Genetic Parameter Identification of the DoyleFuller-Newman Model from Experimental Cycling of a LiFePO4 Battery". American Control Conference.

[12] Forgez, C., Do, D. V., Friedrich, G., Morcrette, M., and Delacourt, C., 2010. "Thermal modeling of a cylindrical lifepo4/graphite lithium-ion battery". Journal of Power Sources, 195(9), pp. $2961-8$.

[13] Vandenberghe, L., Boyd, S., and Wu, S.-P., 1998. "Determinant Maximization with Linear Matrix Inequality Constraints". SIAM Journal on Matrix Analysis and Applications, 19(2), pp. $499-533$. 